\title{
SPECIAL SUBGROUPS OF BIANCHI GROUPS
}

\author{
MICHELLE CHU
}

\begin{abstract}
We determine C-special subgroups of the Bianchi groups of index bounded above by 120 by effectivising the arguments of Agol-Long-Reid. These subgroups are congruence of level 2 or 4 and retract to the free group on two generators. As a consequence, we find a C-special 20-sheeted cover of the figure-eight knot complement. We also determine C-special congruence subgroups for a family of cocompact arithmetic Kleinian groups.
\end{abstract}

\section{INTRODUCTION}

A 3-manifold $M$ is said to have a virtual property if some finite-sheeted cover of $M$ has that property. Similarly, a group $\Gamma$ has a virtual property if some finite-index subgroup of $\Gamma$ has that property. It was shown in CLR97 that non-compact finite volume 3-manifolds virtually contain closed embedded essential surfaces. More recent progress in 3-manifold theory has determined the virtual Haken and the virtual fibering conjectures for all finite volume hyperbolic 3-manifolds, asserting that every finite volume hyperbolic 3-manifold virtually contains an embedded essential surface, and furthermore, is virtually a surface bundle over the circle. These results are implied by a stronger theorem which states that the fundamental groups of finite volume 3-manifolds are virtually special (see Ago13 for the closed case and Wis11 or GM17 for the cusped case). A group is special as in HW08 if it embeds in a right-angled Artin group ( $A$-special) or in a right-angled Coxeter group ( $C$-special). Every right-angled Artin group (RAAG) is a finite-index subgroup of a right-angled Coxeter group (RACG), so every special group embeds in a RACG DJ00. Virtually special groups inherit many nice properties from the RACG. In particular, for fundamental groups of hyperbolic 3-manifolds, virtually special (together with tameness Ago04, CG06 and Canary's covering theorem Can96]) implies LERF and both the virtual Haken and the virtual fibering conjectures.

The goal of this note is to address the following question:

Question 1.1. Given a virtually special group, can one determine a finite-index special subgroup? Can one bound its index?

Prior to Agol's and Wise's results Ago13, Wis11, virtually special was known for several classes 3-manifold groups. Agol, Long, and Reid showed in ALR01 that the Bianchi groups are virtually C-special. Later in BHW11, Bergeron, Haglund, and Wise showed that the fundamental group of an arithmetic hyperbolic manifold of simplest type is virtually C-special. Recently, Question 1.1 was answered for the Seifert-Weber dodecahedral space, which is an arithmetic hyperbolic manifold of simplest type. Spreer and Tillmann constructed in ST17] an A-special cover of the Seifert-Weber dodecahedral space with degree 60 . In this paper we algebraically construct C-special covers of the Bianchi groups. 
Theorem 1.2. Let $m$ be a square-free positive integer and $\mathcal{O}_{m}$ the ring of integers in the quadratid imaginary field $\mathbb{Q}(\sqrt{-m})$. The Bianchi group $\operatorname{PSL}\left(2, \mathcal{O}_{m}\right)$ contains a subgroup $\Delta_{m}$ which embeds in a RACG and has index

$$
\left[\operatorname{PSL}\left(2, \mathcal{O}_{m}\right): \Delta_{m}\right]= \begin{cases}48 & \text { if } m \equiv 1,2 \bmod (4) \\ 120 & \text { if } m \equiv 3 \bmod (8) \\ 72 & \text { if } m \equiv 7 \bmod (8)\end{cases}
$$

where $\Delta_{m}$ is a principal congruence subgroup of level 2 if $m \equiv 1,2 \bmod (4)$ and is otherwise congruence of level 4.

This result relies on making effective the strategy exploited by Agol, Long, and Reid in ALR01. The main idea is to realize quadratic forms associated to the Bianchi groups as sub-forms of the standard form of signature $(6,1)$. The orthogonal group $O(6,1 ; \mathbb{Z})$ contains the reflection group of a right-angled 6 -dimensional hyperbolic Coxeter polytope. In fact, each $\mathbb{H}^{3} / \Delta_{m}$ immerses totally geodesically in this reflection orbifold.

A particular subgroup of a Bianchi group which has attracted much interest is the fundamental group of the figure-eight knot complement. The holonomy representation of the hyperbolic structure is given by Ril75]

$$
\Gamma_{8}:=\left\langle\left(\begin{array}{ll}
1 & 1 \\
0 & 1
\end{array}\right),\left(\begin{array}{cc}
1 & 0 \\
\frac{1+\sqrt{-3}}{2} & 1
\end{array}\right)\right\rangle \subset \operatorname{PSL}\left(2, \mathcal{O}_{3}\right) \text { of index } 12 .
$$

It can be shown that the intersection $\Gamma_{8} \cap \Delta_{3}$ is a subgroup of $\Gamma_{8}$ of index 20 and we get the following corollary.

Corollary 1.3. The figure-eight knot complement has a special finite-sheeted cover of degree 20.

Another consequence of the embedding of $\Delta_{m}$ in a RACG is that $\Delta_{m}$ is virtually RFRS Ago08 (in fact, contains a RFRS subgroup of index $2^{26}$ ) and virtually retracts to its geometrically finite subgroups [LR08.

Proposition 1.4. The subgroup $\Delta_{m}$ retracts onto the free group on 2 generators.

The paper is organized as follows: In $\S 2$ we give some preliminaries on orthogonal groups, congruence subgroups, and right-angled Coxeter groups. We introduce Bianchi groups and their associated quadratic forms in $\S 3$. The proofs of Theorem 1.2 and Proposition 1.4 are in $\S 4$ Finally, we describe some cocompact examples in $\$ 5$.

\section{Preliminaries}

2.1. Orthogonal groups of quadratic forms. Let $f$ be a quadratic form with coefficients in a number field $K$. Let $S_{f}$ be the symmetric matrix associated to $f$. We say that two quadratic forms $f_{1}$ and $f_{2}$ are equivalent over $k$ is there exist some matrix $A \in \mathrm{GL}(n, K)$ such that $A^{t} S_{f_{1}} A=S_{f_{2}}$.

For $K$ a real number field, $\mathcal{O}_{K}$ its ring of integers, and $f$ a quadratic form over $K$ in $n+1$ variables, the Orthogonal group of $f$ is the group

$$
\mathrm{O}(f)=\left\{M \in \mathrm{GL}(n+1, \mathbb{R}) \mid M^{t} S_{Q} M=S_{Q}\right\} .
$$

Now fix $K$ a totally real number field. Consider a quadratic form $Q$ defined on a vector space $V$ over $K$ of dimension $n+1$ such that $Q$ has signature $(n, 1)$ but 
for every non-identity embedding $\sigma: k \hookrightarrow \mathbb{R}, Q^{\sigma}$ is positive-definite. Let $\mathbb{R}^{n, 1}$ be the $(n+1)$-dimensional vector space with symmetric bilinear form

$$
(u, v)=\frac{1}{2}(Q(u+v)-Q(u)-Q(v))
$$

and symmetric matrix $S_{Q}$ (the symmetric matrix in $\operatorname{GL}(n+1, \mathbb{R})$ with $Q(v)=$ $\left.v^{t} S_{Q} v\right)$.

Let $\left\{x_{0}, x_{1}, \ldots, x_{n}\right\}$ be an orthogonal basis for $Q$ with $Q\left(x_{0}\right)<0$ and $Q\left(x_{i}\right)>0$ for each $i \geq 1$. The vectors $v \in \mathbb{R}^{n, 1}$ with $Q(v)=1$ form an $n$-dimensional hyperboloid $\mathcal{C}$ consisting of a positive sheet $\mathcal{C}^{+}=\left\{v \in \mathcal{C} \mid v_{0}>0\right\}$ and a negative sheet $\mathcal{C}^{-}=\left\{v \in \mathcal{C} \mid v_{0}<0\right\}$. The hyperboloid model of hyperbolic space $\mathbb{H}^{n}$ is identified with $S^{+}$. The isometires of $\mathbb{H}^{n}$ are the orthogonal transformations of $\mathbb{R}^{n, 1}$.

Then $\mathrm{O}(Q ; \mathbb{R})$ is the isometry group which preserves $\mathcal{C}$. The index two subgroup which preserves the positive sheet $\mathcal{C}^{+}$is called $\mathrm{O}^{+}(Q ; \mathbb{R})$ (i.e. preserves the sign of $\left.v_{0}\right)$. This subgroup $\mathrm{O}^{+}(Q ; \mathbb{R})$ is identified with the full group of isometries of $\mathbb{H}^{n}$ and has two connected components, whose elements either preserve or reverse orientation. The orientation preserving isometry group of $\mathbb{H}^{n}$ is identified with the component of matrices with determinant $1, \mathrm{SO}^{+}(Q ; \mathbb{R})$.

The subgroup $\mathrm{O}^{+}\left(Q, \mathcal{O}_{K}\right)=\mathrm{O}^{+}(Q, \mathbb{R}) \cap \mathrm{GL}\left(n+1, \mathcal{O}_{K}\right)$ (or $\mathrm{SO}^{+}\left(Q, \mathcal{O}_{K}\right)=$ $\left.\mathrm{SO}^{+}(Q, \mathbb{R}) \cap \mathrm{GL}\left(n+1, \mathcal{O}_{K}\right)\right)$ of integral isometries is a lattice, discrete subgroup of finite covolume, in $\mathrm{O}(Q ; \mathbb{R})$ (or $\mathrm{SO}(Q ; \mathbb{R})$ ). Any lattice commensurable with such an $\mathrm{O}\left(Q ; \mathcal{O}_{K}\right)$ or $\mathrm{SO}\left(Q ; \mathcal{O}_{K}\right)$ is called an arithmetic lattice of simplest type.

2.2. Congruence subgroups. If $\mathscr{I}$ is an ideal in a ring $R$ and $\Delta \leq \operatorname{SL}(n, R)$, the principal congruence subgroup of level $(\mathscr{I})$ in $\Delta$ is the subgroup of matrices $M \in \Delta$ which can be written as $M=I+\mathscr{I} A$ where $A \in \mathrm{GL}(n, R)$. We denote this subgroup by $\Delta_{(\mathscr{I})}$.

2.3. Right-angled hyperbolic polytope. A polytope in $n$-dimensional hyperbolic space $\mathbb{H}^{n}$ is a convex polyhedron with finitely many actual and ideal vertices which is the convex hull of its vertices. A polytope is a Coxeter polytope if the dihedral angle between any two adjacent sides is either 0 or $\pi / k$ for some integer $k \geq 2$. A Coxeter polytope is right-angled if all the dihedral angles are either 0 or $\pi / 2$.

The group generated by the reflections in the sides of a Coxeter polytope is a hyperbolic Coxeter group and is a discrete subgroup of $\operatorname{Isom}\left(\mathbb{H}^{n}\right)$. The group generated by reflections in the sides of a right-angled polytope is also right-angled Coxeter group, denoted $R A C G$.

Let $F_{n}$ be the diagonal quadratic form

$$
F_{n}:=-x_{0}^{2}+x_{1}^{2}+\cdots+x_{n}^{2} .
$$

Theorem 2.1 ([ERT12, Theorem 2.1]). For $2 \leq n \leq 7, \mathrm{O}^{+}\left(F_{n} ; \mathbb{Z}\right)_{(2)}$ is a $R A C G$. It is the reflection group of an all-right hyperbolic polytope of dimension $n$, and $\mathrm{SO}^{+}\left(F_{n} ; \mathbb{Z}\right)_{(2)}$ is its index 2 subgroup of orientation preserving isometries. 


\section{BIANCHI GROUPS AND QUADRATIC FORMS}

The Bianchi groups are the arithmetic Kleinian groups $\operatorname{PSL}\left(2, \mathcal{O}_{m}\right)$ where $m$ is a positive square-free integer and

$$
\mathcal{O}_{m}= \begin{cases}\mathbb{Z}[\sqrt{-m}] & m \equiv 1,2 \quad \bmod (4) \\ \mathbb{Z}\left[\frac{1+\sqrt{-m}}{2}\right] & m \equiv 3 \quad \bmod (4)\end{cases}
$$

is the ring of integers in the field $\mathbb{Q}(\sqrt{-m})$. Any non-cocompact arithmetic lattice in $\operatorname{Isom}^{+}\left(\mathbb{H}^{3}\right)$ is commensurable to some Bianchi group (see e.g. [MR03, §8.2]).

3.1. The Bianchi groups and quadratic forms. We now give a precise relationship between Bianchi groups and orthogonal groups of quadratic forms following [JM96, §2] and [EGM98, §1.3].

For $m$ square-free integer, define the quadratic forms

$$
Q_{m}\left(x_{0}, x_{1}, x_{2}, x_{3}\right):= \begin{cases}2 x_{0} x_{1}+2 x_{2}^{2}+2 m x_{3}^{2} & \text { if } m \equiv 1,2 \bmod 4 \\ 2 x_{0} x_{1}+2 x_{2}^{2}+2 x_{2} x_{3}+\frac{m+1}{2} x_{3}^{2} & \text { if } m \equiv 3 \bmod 4\end{cases}
$$

with corresponding symmetric matrices

$$
S_{m_{1,2}}=\left(\begin{array}{cccc}
0 & 1 & 0 & 0 \\
1 & 0 & 0 & 0 \\
0 & 0 & 2 & 0 \\
0 & 0 & 0 & 2 m
\end{array}\right) \text { and } S_{m_{3}}=\left(\begin{array}{cccc}
0 & 1 & 0 & 0 \\
1 & 0 & 0 & 0 \\
0 & 0 & 2 & 1 \\
0 & 0 & 1 & \frac{m+1}{2}
\end{array}\right)
$$

For $m \equiv 1,2 \bmod 4$, define the homomorphism

$$
\varphi_{m}: \operatorname{PGL}\left(2, \mathcal{O}_{m}\right) \rightarrow \mathrm{SO}\left(Q_{m} ; \mathbb{Z}\right)
$$

by sending $\alpha=\left(\begin{array}{ll}a_{0}+a_{1} \sqrt{-m} & b_{0}+b_{1} \sqrt{-m} \\ c_{0}+c_{1} \sqrt{-m} & d_{0}+d_{1} \sqrt{-m}\end{array}\right)$ to $(\operatorname{det}(\alpha))^{-1}$ times

$$
\left(\begin{array}{ccc}
d_{0}^{2}+m d_{1}^{2} & -c_{0}^{2}-m c_{1}^{2} & 2\left(c_{0} d_{0}+m c_{1} d_{1}\right) \\
-b_{0}^{2}-m b_{1}^{2} & a_{0}^{2}+m a_{1}^{2} & -2\left(a_{0} b_{0}+m a_{1} b_{1}\right) \\
b_{0} d_{0}+m b_{1} d_{1} & -a_{0} c_{0}-m a_{1} c_{1} & b_{0} c_{0}+m b_{1} c_{1}+a_{0} d_{0}+m a_{1} d_{1} \\
b_{1} d_{0}-b_{0} d_{1} & a_{0} c_{1}-a_{1} c_{0} & b_{1} c_{0}-b_{0} c_{1}+a_{1} d_{0}-a_{0} d_{1} \\
& & -2 m\left(c_{1} d_{0}-c_{0} d_{1}\right) \\
& & 2 m\left(a_{1} b_{0}-a_{0} b_{1}\right) \\
& m\left(b_{1} c_{0}-b_{0} c_{1}-a_{1} d_{0}+a_{0} d_{1}\right) \\
& -b_{0} c_{0}-m b_{1} c_{1}+a_{0} d_{0}+m a_{1} d_{1}
\end{array}\right) .
$$

The kernel of this homomorphism is $\mathbb{Q}^{\times} I$, so it restricts to an injection

$$
\operatorname{PSL}\left(2, \mathcal{O}_{m}\right) \hookrightarrow \mathrm{SO}^{+}\left(Q_{m} ; \mathbb{Z}\right)
$$

whose image is the spinorial group [JM96.

For $m \equiv 3 \bmod 4$, let $m=4 k-1$ and define the homomorphism

$$
\varphi_{m}: \operatorname{PGL}\left(2, \mathcal{O}_{m}\right) \rightarrow \mathrm{SO}\left(Q_{m} ; \mathbb{Z}\right)
$$


by sending $\alpha=\left(\begin{array}{ll}a_{0}+a_{1} \frac{1+\sqrt{-m}}{2} & b_{0}+b_{1} \frac{1+\sqrt{-m}}{2} \\ c_{0}+c_{1} \frac{1+\sqrt{-m}}{2} & d_{0}+d_{1} \frac{1+\sqrt{-m}}{2}\end{array}\right)$ to $(\operatorname{det}(\alpha))^{-1}$ times

$$
\left(\begin{array}{ccc}
d_{0}^{2}+d_{1} d_{0}+k d_{1}^{2} & -c_{0}^{2}-c_{1} c_{0}-k c_{1}^{2} & 2 c_{0} d_{0}+c_{1} d_{0}+c_{0} d_{1}+2 k c_{1} d_{1} \\
-b_{0}^{2}-b_{1} b_{0}-k b_{1}^{2} & a_{0}^{2}+a_{1} a_{0}+k a_{1}^{2} & -2 a_{0} b_{0}-a_{1} b_{0}-a_{0} b_{1}-2 k a_{1} b_{1} \\
b_{0} d_{0}+b_{1} d_{0}+k b_{1} d_{1} & -a_{0} c_{0}-a_{1} c_{0}-k a_{1} c_{1} & b_{0} c_{0}+b_{1} c_{0}+k b_{1} c_{1}+a_{0} d_{0}+a_{1} d_{0}+k a_{1} d_{1} \\
b_{0} d_{1}-b_{1} d_{0} & a_{1} c_{0}-a_{0} c_{1} & -b_{1} c_{0}+b_{0} c_{1}-a_{1} d_{0}+a_{0} d_{1} \\
c_{0} d_{0}+2 k c_{1} d_{0}-2 k c_{0} d_{1}+c_{0} d_{1}+k c_{1} d_{1} \\
-a_{0} b_{0}-2 k a_{1} b_{0}+2 k a_{0} b_{1}-a_{0} b_{1}-k a_{1} b_{1} \\
b_{0} c_{0}-k b_{1} c_{0}+b_{1} c_{0}+k b_{0} c_{1}+k b_{1} c_{1}+k a_{1} d_{0}-k a_{0} d_{1} \\
-b_{0} c_{0}-b_{1} c_{0}-k b_{1} c_{1}+a_{0} d_{0}+a_{0} d_{1}+k a_{1} d_{1}
\end{array}\right) .
$$

The kernel of this homomorphism is $\mathbb{Q}^{\times} I$, so it restricts to an injection

$$
\operatorname{PSL}\left(2, \mathcal{O}_{m}\right) \hookrightarrow \mathrm{SO}^{+}\left(Q_{m} ; \mathbb{Z}\right)
$$

3.2. Congruence subgroups of Bianchi groups. If $\mathscr{I}$ is an ideal in $\mathcal{O}_{m}$, the quotient $\operatorname{PSL}\left(2, \mathcal{O}_{m}\right) / \operatorname{PSL}\left(2, \mathcal{O}_{m}\right)(\mathscr{I})$ of $\operatorname{PSL}\left(2, \mathcal{O}_{m}\right)$ by the principal congruence subgroup of level $\mathscr{I}$ is isomorphic to $\operatorname{PSL}\left(2, \mathcal{O}_{m} / \mathscr{I}\right)$. The norm of $\mathscr{I}$ is given by $N(\mathscr{I})=\left|\mathcal{O}_{m} / \mathscr{I}\right|$ and is multiplicative $N(\mathscr{I} \mathscr{J})=N(\mathscr{I}) N(\mathscr{J})$.

This gives a formula for the index $\operatorname{PSL}\left(2, \mathcal{O}_{m}\right)(\mathscr{I})$ using a decomposition of $\mathscr{I}$ into powers of prime ideals $\mathscr{I}=\mathcal{P}_{1}^{j_{1}} \cdots \mathcal{P}_{r}^{j_{r}}$ (see [Dic01]). We have

$$
\left[\operatorname{PSL}\left(2, \mathcal{O}_{m}\right): \operatorname{PSL}\left(2, \mathcal{O}_{m}\right)(\mathscr{I})\right]= \begin{cases}6 & \text { when } N(\mathscr{I})=2 \\ N(\mathscr{I})^{3} \prod_{\mathcal{P} \mid \mathscr{I}}\left(1-\frac{1}{N(\mathscr{I})^{2}}\right) & \text { when } 2 \in \mathscr{I} \\ \frac{N(\mathscr{I})^{3}}{2} \prod_{\mathcal{P} \mid \mathscr{I}}\left(1-\frac{1}{N(\mathscr{I})^{2}}\right) & \text { otherwise }\end{cases}
$$

For a rational prime $p$ there are three possibilities for the decomposition of the ideal $p \mathcal{O}_{m}$ in $\mathcal{O}_{m}$ :

$$
p \mathcal{O}_{m}= \begin{cases}\mathcal{P}^{2} & p \text { is ramified and } N(\mathcal{P})=p \\ \mathcal{P} & p \text { is inert and } N(\mathcal{P})=p^{2} \\ \mathcal{P}_{1} \mathcal{P}_{2} & p \text { is split and } N\left(\mathcal{P}_{1}\right)=N\left(\mathcal{P}_{2}\right)=p .\end{cases}
$$

Therefore since 2 is ramified when $m \equiv 1,2 \bmod 4$, is inert when $m \equiv 3 \bmod 4$, and is split when $m \equiv 7 \bmod 4$ we have

$$
\left[\operatorname{PSL}\left(2, \mathcal{O}_{m}\right): \operatorname{PSL}\left(2, \mathcal{O}_{m}\right)_{(2)}\right]= \begin{cases}48 & \text { if } m \equiv 1,2 \bmod 4 \\ 60 & \text { if } m \equiv 3 \bmod 8 \\ 36 & \text { if } m \equiv 7 \bmod 8\end{cases}
$$

\section{Proof of Theorem 1.2}

4.1. The special subgroup for $m \equiv 1,2 \bmod 4$. By Equation 3.6 when $m \equiv 1,2$ $\bmod 4$ we have

$$
\left[\operatorname{PSL}\left(2, \mathcal{O}_{m}\right): \operatorname{PSL}\left(2, \mathcal{O}_{m}\right)_{(2)}\right]=48 .
$$

Proposition 4.1. Let $m \equiv 1,2 \bmod 4$ be square-free positive. The principal con-

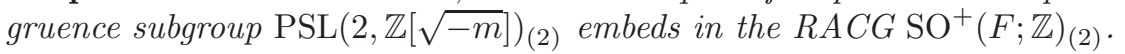


Proof. Let $P_{m}:=Q_{m} \oplus\langle 2 m, 2 m, 2 m\rangle$. The group $\mathrm{SO}^{+}\left(Q_{m} ; \mathbb{Z}\right)$ is naturally a subgroup of $\mathrm{SO}^{+}\left(P_{m} ; \mathbb{Z}\right)$.

By Lagrange's 4-square theorem, write $m=w^{2}+x^{2}+y^{2}+z^{2}$, a sum of four squares. Consider the $7 \times 7$ matrices

$$
A_{m}=\left(\begin{array}{ccccccc}
\frac{1}{2} & -\frac{1}{2} & 0 & 0 & 0 & 0 & 0 \\
\frac{1}{2} & \frac{1}{2} & 0 & 0 & 0 & 0 & 0 \\
0 & 0 & 1 & 0 & 0 & 0 & 0 \\
0 & 0 & 0 & w & -x & -y & -z \\
0 & 0 & 0 & x & w & z & -y \\
0 & 0 & 0 & y & -z & w & x \\
0 & 0 & 0 & z & y & -x & w
\end{array}\right)
$$

with inverse

$$
A_{m}^{-1}=\left(\begin{array}{ccccccc}
1 & 1 & 0 & 0 & 0 & 0 & 0 \\
-1 & 1 & 0 & 0 & 0 & 0 & 0 \\
0 & 0 & 1 & 0 & 0 & 0 & 0 \\
0 & 0 & 0 & \frac{w}{m} & \frac{x}{m} & \frac{y}{m} & \frac{z}{m} \\
0 & 0 & 0 & -\frac{x}{m} & \frac{w}{m} & -\frac{z}{m} & \frac{y}{m} \\
0 & 0 & 0 & -\frac{y}{m} & \frac{z}{m} & \frac{w}{m} & -\frac{x}{m} \\
0 & 0 & 0 & -\frac{z}{m} & -\frac{y}{m} & \frac{x}{m} & \frac{w}{m}
\end{array}\right) .
$$

Let $S_{F}$ be the diagonal matrix associated to $F_{6}$ and $S_{P_{m}}$ the symmetric matrix associated to $\frac{1}{2} P_{m}$ :

$$
\begin{aligned}
S_{F} & =\left(\begin{array}{ccccccc}
-1 & 0 & 0 & 0 & 0 & 0 & 0 \\
0 & 1 & 0 & 0 & 0 & 0 & 0 \\
0 & 0 & 1 & 0 & 0 & 0 & 0 \\
0 & 0 & 0 & 1 & 0 & 0 & 0 \\
0 & 0 & 0 & 0 & 1 & 0 & 0 \\
0 & 0 & 0 & 0 & 0 & 1 & 0 \\
0 & 0 & 0 & 0 & 0 & 0 & 1
\end{array}\right) \\
S_{P_{m}} & =\left(\begin{array}{cccccccc}
0 & \frac{1}{2} & 0 & 0 & 0 & 0 & 0 \\
\frac{1}{2} & 0 & 0 & 0 & 0 & 0 & 0 \\
0 & 0 & 1 & 0 & 0 & 0 & 0 \\
0 & 0 & 0 & m & 0 & 0 & 0 \\
0 & 0 & 0 & 0 & m & 0 & 0 \\
0 & 0 & 0 & 0 & 0 & m & 0 \\
0 & 0 & 0 & 0 & 0 & 0 & m
\end{array}\right) .
\end{aligned}
$$

Then $A_{m}^{t} S_{F} A_{m}=S_{P_{m}}$. Since $A_{m}$ has determinant $-m^{2} / 2$, it is in $\operatorname{GL}(7, \mathbb{Q})$ and the forms $F_{6}$ and $P_{m}$ are equivalent over $\mathbb{Q}$ and thus $A_{m} \mathrm{SO}^{+}\left(P_{m} ; \mathbb{Q}\right) A_{m}^{-1}=$ $\mathrm{SO}^{+}\left(F_{6} ; \mathbb{Q}\right)$. Therefore, $A_{m} \mathrm{SO}^{+}\left(Q_{m} ; \mathbb{Q}\right) A_{m}^{-1} \subset \mathrm{SO}^{+}\left(F_{6} ; \mathbb{Q}\right)$.

A matrix $N$ in $\operatorname{PSL}\left(2, \mathcal{O}_{m}\right)_{(2)}$ has form

$$
\left(\begin{array}{cc}
2 a_{0}+1+2 a_{1} \sqrt{-m} & 2 b_{0}+2 b_{1} \sqrt{-m} \\
2 c_{0}+2 c_{1} \sqrt{-m} & 2 d_{0}+1+2 d_{1} \sqrt{-m}
\end{array}\right)
$$


and from Equation 3.4 its image in $\varphi_{m}$ is given by

$$
\left(\begin{array}{cc}
4\left(d_{0}^{2}+d_{0}+m d_{1}^{2}\right)+1 & -4\left(c_{0}^{2}+m c_{1}^{2}\right) \\
-4\left(b_{0}^{2}+m b_{1}^{2}\right) & 4\left(a_{0}^{2}+a_{0}+m a_{1}^{2}\right)+1 \\
2\left(2 d_{0} b_{0}+b_{0}+2 m b_{1} d_{1}\right) & -2\left(2 a_{0} c_{0}+c_{0}+2 m a_{1} c_{1}\right) \\
2\left(2 d_{0} b_{1}+b_{1}-2 b_{0} d_{1}\right) & -2\left(2 a_{1} c_{0}-2 a_{0} c_{1}-c_{1}\right) \\
4\left(2 d_{0} c_{0}+c_{0}+2 m c_{1} d_{1}\right) \\
-4\left(2 a_{0} b_{0}+b_{0}+2 m a_{1} b_{1}\right) \\
2\left(2 d_{0} a_{0}+a_{0}+2 b_{0} c_{0}+2 m b_{1} c_{1}+d_{0}+2 m a_{1} d_{1}\right)+1 \\
2\left(2 d_{0} a_{1}+a_{1}+2 b_{1} c_{0}-2 b_{0} c_{1}-2 a_{0} d_{1}-d_{1}\right) \\
-4 m\left(2 d_{0} c_{1}+c_{1}-2 c_{0} d_{1}\right) \\
4 m\left(2 a_{1} b_{0}-2 a_{0} b_{1}-b_{1}\right) \\
2 m\left(-2 d_{0} a_{1}-a_{1}+2 b_{1} c_{0}-2 b_{0} c_{1}+2 a_{0} d_{1}+d_{1}\right) \\
1-2\left(-2 d_{0} a_{0}-a_{0}+2 b_{0} c_{0}+2 m b_{1} c_{1}-d_{0}-2 m a_{1} d_{1}\right)
\end{array}\right) .
$$

Let $N^{\prime}$ be

$$
\left(\begin{array}{cc}
\varphi_{m}(N) & 0_{4 \times 3} \\
0_{3 \times 4} & I_{3 \times 3}
\end{array}\right) \in \mathrm{SO}^{+}\left(P_{m} ; \mathbb{Z}\right)
$$

It is now an easy check to see that $A_{m} N^{\prime} A_{m}^{-1} \in \mathrm{SO}^{+}\left(F_{6} ; \mathbb{Q}\right)$ and in fact $A_{m} N^{\prime} A_{m}^{-1} \equiv$ $I \bmod (2)$. So $A_{m} N^{\prime} A_{m}^{-1} \in \mathrm{SO}^{+}(F ; \mathbb{Z})_{(2)}$.

Remark 4.2. From Equation 4.6 we see that

$$
\varphi_{m}\left(\operatorname{PSL}\left(2, \mathcal{O}_{m}\right)\right)_{(4)} \leq \varphi_{m}\left(\operatorname{PSL}\left(2, \mathcal{O}_{m}\right)_{(2)}\right) \leq \varphi_{m}\left(\operatorname{PSL}\left(2, \mathcal{O}_{m}\right)\right)_{(2)} .
$$

Remark 4.3. In the ring of integers $\mathcal{O}_{m}$ for the number field $\mathbb{Q}(\sqrt{-m}),(2)$ is not always a prime ideal. One might ask whether a congruence subgroup with level a prime over 2 suffices to embed in a RACG. Unfortunately, using the methods above, a prime over 2 is not enough. Consider as an example the case of $m=1$. The ideal $(2)$ ramifies as $(1+i)^{2}$. However, the principal congruence subgroup $\operatorname{PSL}(2, \mathbb{Z}[i])_{(1+i)}$ is not contained in $\mathrm{SO}^{+}(F ; \mathbb{Z})_{(2)}$ via the map $\varphi_{1}$ and the conjugation by $A_{m_{1,2}}$. Indeed if we write $1=1^{2}+0+0+0$ (i.e. $w=1, x=y=z=0$ in the definition of $A_{m_{1,2}}$ in Equation 4.2, the element

$$
A_{1}^{-1} \varphi_{1}\left(\left(\begin{array}{cc}
1 & 1+i \\
0 & 1
\end{array}\right)\right) A_{1}=\left(\begin{array}{ccccccc}
2 & 1 & 1 & 1 & 0 & 0 & 0 \\
-1 & 0 & -1 & -1 & 0 & 0 & 0 \\
1 & 1 & 1 & 0 & 0 & 0 & 0 \\
1 & 1 & 0 & 1 & 0 & 0 & 0 \\
0 & 0 & 0 & 0 & 1 & 0 & 0 \\
0 & 0 & 0 & 0 & 0 & 1 & 0 \\
0 & 0 & 0 & 0 & 0 & 0 & 1
\end{array}\right)
$$

does not reduce to the identity modulo 2 .

\subsection{The special subgroup for $m \equiv 3 \bmod 4$.}

Proposition 4.4. Let $m \equiv 3 \bmod 4$ be square-free positive. The principal congruence subgroup $\operatorname{PSL}(2, \mathbb{Z}[\sqrt{-m}])_{(2)}$ has an index 2 subgroup $\Delta_{m}$ which embeds in the $R A C G \mathrm{SO}^{+}(F ; \mathbb{Z})_{(2)}$ and with

$$
\left[\operatorname{PSL}\left(2, \mathcal{O}_{m}\right): \Delta_{m}\right]=\left\{\begin{array}{lll}
120 & \text { if } m \equiv 3 & \bmod 8 \\
72 & \text { if } m \equiv 7 & \bmod 8
\end{array}\right.
$$


Proof. Let $P_{m}:=Q_{m} \oplus\langle 2 m, 2 m, 2 m\rangle$. The group $\mathrm{SO}^{+}\left(Q_{m} ; \mathbb{Z}\right)$ is naturally a subgroup of $\mathrm{SO}^{+}\left(P_{m} ; \mathbb{Z}\right)$.

By Lagrange's 4-square theorem, write $m=w^{2}+x^{2}+y^{2}+z^{2}$, a sum of four squares. Consider the $7 \times 7$ matrices

$$
A_{m_{3}}=\left(\begin{array}{ccccccc}
\frac{1}{2} & -\frac{1}{2} & 0 & 0 & 0 & 0 & 0 \\
\frac{1}{2} & \frac{1}{2} & 0 & 0 & 0 & 0 & 0 \\
0 & 0 & 1 & \frac{1}{2} & 0 & 0 & 0 \\
0 & 0 & 0 & -\frac{w}{2} & -x & -y & -z \\
0 & 0 & 0 & -\frac{x}{2} & w & z & -y \\
0 & 0 & 0 & -\frac{y}{2} & -z & w & x \\
0 & 0 & 0 & -\frac{z}{2} & y & -x & w
\end{array}\right)
$$

with inverse

$$
A_{m_{3}}^{-1}=\left(\begin{array}{ccccccc}
1 & 1 & 0 & 0 & 0 & 0 & 0 \\
-1 & 1 & 0 & 0 & 0 & 0 & 0 \\
0 & 0 & 1 & \frac{w}{m} & \frac{x}{m} & \frac{y}{m} & \frac{z}{m} \\
0 & 0 & 0 & -\frac{2 w}{m} & -\frac{2 x}{m} & -\frac{2 y}{m} & -\frac{2 z}{m} \\
0 & 0 & 0 & -\frac{x}{m} & \frac{w}{m} & -\frac{z}{m} & \frac{y}{m} \\
0 & 0 & 0 & -\frac{y}{m} & \frac{z}{m} & \frac{w}{m} & -\frac{x}{m} \\
0 & 0 & 0 & -\frac{z}{m} & -\frac{y}{m} & \frac{x}{m} & \frac{w}{m}
\end{array}\right) .
$$

Let $S_{F}$ be the diagonal matrix associated to $F_{6}$ (see Equation 4.4) and $S_{P_{m}}$ the symmetric matrix associated to $\frac{1}{2} P_{m}$ :

$$
\left(\begin{array}{ccccccc}
0 & \frac{1}{2} & 0 & 0 & 0 & 0 & 0 \\
\frac{1}{2} & 0 & 0 & 0 & 0 & 0 & 0 \\
0 & 0 & 1 & \frac{1}{2} & 0 & 0 & 0 \\
0 & 0 & \frac{1}{2} & \frac{m+1}{4} & 0 & 0 & 0 \\
0 & 0 & 0 & 0 & m & 0 & 0 \\
0 & 0 & 0 & 0 & 0 & m & 0 \\
0 & 0 & 0 & 0 & 0 & 0 & m
\end{array}\right)
$$

Then $A_{m}^{t} S_{F} A_{m}=S_{P_{m}}$. Since $A_{m}$ has determinant $-m^{2} / 4$, it is in $\operatorname{GL}(7, \mathbb{Q})$ and the forms $F_{6}$ and $P_{m}$ are equivalent over $\mathbb{Q}$ and thus $A_{m} \mathrm{SO}^{+}\left(P_{m} ; \mathbb{Q}\right) A_{m}^{-1}=$ $\mathrm{SO}^{+}\left(F_{6} ; \mathbb{Q}\right)$. Therefore, $A_{m} \mathrm{SO}^{+}\left(Q_{m} ; \mathbb{Q}\right) A_{m}^{-1} \subset \mathrm{SO}^{+}\left(F_{6} ; \mathbb{Q}\right)$.

A matrix $N$ in $\operatorname{PSL}\left(2, \mathcal{O}_{m}\right)_{(2)}$ has form

$$
\left(\begin{array}{cc}
2 a_{0}+1+2 a_{1} \frac{1+\sqrt{-m}}{2} & 2 b_{0}+2 b_{1} \frac{1+\sqrt{-m}}{2} \\
2 c_{0}+2 c_{1} \frac{1+\sqrt{-m}}{2} & 2 d_{0}+1+2 d_{1} \frac{1+\sqrt{-m}}{2}
\end{array}\right),
$$


and from Equation 3.5 its image in $\varphi_{m}$ is given by

$$
\left(\begin{array}{cc}
2 d_{1}+4\left(d_{0}^{2}+d_{1} d_{0}+d_{0}+k d_{1}^{2}\right)+1 & -4\left(c_{0}^{2}+c_{1} c_{0}+k c_{1}^{2}\right) \\
-4\left(b_{0}^{2}+b_{1} b_{0}+k b_{1}^{2}\right) & 2 a_{1}+4\left(a_{0}^{2}+a_{1} a_{0}+a_{0}+k a_{1}^{2}\right)+1 \\
2\left(b_{0}+b_{1}\right)+4\left(d_{0} b_{0}+b_{1} d_{0}+k b_{1} d_{1}\right) & -2 c_{0}-4\left(a_{0} c_{0}+a_{1} c_{0}+k a_{1} c_{1}\right) \\
-2 b_{1}-4\left(d_{0} b_{1}-b_{0} d_{1}\right) & -2 c_{1}+4\left(a_{1} c_{0}-a_{0} c_{1}\right) \\
2\left(c_{1}\right)+4\left(2 d_{0} c_{0}+d_{1} c_{0}+c_{0}+c_{1} d_{0}+2 k c_{1} d_{1}\right) \\
-2 b_{1}-4\left(2 a_{0} b_{0}+a_{1} b_{0}+b_{0}+a_{0} b_{1}+2 k a_{1} b_{1}\right) \\
2\left(d_{1}-a_{1}\right)-4\left(d_{0} a_{1}+b_{1} c_{0}-b_{0} c_{1}-a_{0} d_{1}\right) \\
2 c_{0}+4\left(d_{0} c_{0}-2 k d_{1} c_{0}+d_{1} c_{0}+k c_{1}+2 k c_{1} d_{0}+k c_{1} d_{1}\right)+1 \\
-2\left(b_{0}+b_{1}\right)-4\left(a_{0} b_{0}+2 k a_{1} b_{0}-k b_{1}-2 k a_{0} b_{1}+a_{0} b_{1}+k a_{1} b_{1}\right) \\
2\left(k a_{1}-k d_{1}\right)+4\left(b_{0} c_{0}+b_{1} c_{0}\right)+4 k\left(d_{0} a_{1}-b_{1} c_{0}+b_{0} c_{1}+b_{1} c_{1}-a_{0} d_{1}\right) \\
1+2\left(a_{0}+d_{0}+d_{1}\right)-4\left(-d_{0} a_{0}-d_{1} a_{0}+b_{0} c_{0}+b_{1} c_{0}+k b_{1} c_{1}-k a_{1} d_{1}\right)
\end{array}\right) .
$$

Note that $N$ has determinant 1 . This implies

$$
\begin{gathered}
1+2 a_{0}+a_{1}-4 b_{0} c_{0}-2 b_{1} c_{0}-2 b_{0} c_{1}-2 b_{1} c_{1}+2 d_{0}+4 a_{0} d_{0}+2 a_{1} d_{0} \\
+d_{1}+2 a_{0} d_{1}+4 b_{1} c_{1} k-4 a_{1} d_{1} k=1 \\
\text { and } a_{1}-2 b_{1} c_{0}-2 b_{0} c_{1}-2 b_{1} c_{1}+2 a_{1} d_{0}+d_{1}+2 a_{0} d_{1}+2 a_{1} d_{1}=0
\end{gathered}
$$

so $a_{1} \equiv d_{1} \bmod 2$.

Let $N^{\prime}$ be

$$
\left(\begin{array}{cc}
\varphi_{m}(N) & 0_{4 \times 3} \\
0_{3 \times 4} & I_{3 \times 3}
\end{array}\right) \in \mathrm{SO}^{+}\left(P_{m} ; \mathbb{Z}\right)
$$

It is now also true as in the case of $m \equiv 1,2 \bmod 4$ in Proposition 4.1 that $A_{m} N^{\prime} A_{m}^{-1} \in \mathrm{SO}^{+}\left(F_{6} ; \mathbb{Q}\right)$. However, it requires some clever substitutions using Equation 4.12.

Unfortunately, it is not always the case that $A_{m} N^{\prime} A_{m}^{-1} \equiv I \bmod (2)$, indeed

$$
A_{m} N^{\prime} A_{m}^{-1} \equiv\left(\begin{array}{ccccccc}
1 & 0 & b_{1}+c_{1} & w \beta & x \beta & y \beta & z \beta \\
0 & 1 & \beta & w \beta & x \beta & y \beta & z \beta \\
\beta & \beta & 1 & 0 & 0 & 0 & 0 \\
w \beta & w \beta & 0 & 1 & 0 & 0 & 0 \\
x \beta & x \beta & 0 & 0 & 1 & 0 & 0 \\
y \beta & y \beta & 0 & 0 & 0 & 1 & 0 \\
z \beta & z \beta & 0 & 0 & 0 & 0 & 1
\end{array}\right) \bmod 2
$$

where $\beta=b_{1}+c_{1}$. However, consider the subgroup of $\operatorname{PSL}\left(2, \mathcal{O}_{m}\right)_{(2)}$ of index 2 given by

$$
\Delta_{m}:=\left\{\left(\begin{array}{cc}
2 a_{0}+1+2 a_{1} \frac{1+\sqrt{-m}}{2} & 2 b_{0}+2 b_{1} \frac{1+\sqrt{-m}}{2} \\
2 c_{0}+2 c_{1} \frac{1+\sqrt{-m}}{2} & 2 d_{0}+1+2 d_{1} \frac{1+\sqrt{-m}}{2}
\end{array}\right): b_{1} \equiv c_{1} \quad \bmod 2\right\} .
$$

Then for $N \in \Delta_{m} A_{m_{i}} N^{\prime} A_{m_{i}}^{-1} \in \mathrm{SO}^{+}(F ; \mathbb{Z})_{(2)}$ and the proposition follows from Equation 3.6.

Remark 4.5. From Equation 4.11 and Equation 4.14 we see that

$$
\varphi_{m}\left(\operatorname{PSL}\left(2, \mathcal{O}_{m}\right)\right)_{(4)} \leq \varphi_{m}\left(\operatorname{PSL}\left(2, \mathcal{O}_{m}\right)_{(2)}\right) \leq \varphi_{m}\left(\operatorname{PSL}\left(2, \mathcal{O}_{m}\right)\right)_{(2)}
$$

and

$$
\operatorname{PSL}\left(2, \mathcal{O}_{m}\right)_{(4)} \leq \Delta_{m} \leq \operatorname{PSL}\left(2, \mathcal{O}_{m}\right)_{(2)}
$$

so $\Delta_{m}$ is congruence of level 4 , but not principal congruence. 
4.3. Virtual retracts and the RFRS condition. Let $C$ be a hyperbolic rightangled Coxeter polytope of dimension $n>2$, say in $\mathbb{H}^{n}$. Let $\Gamma$ be the group generated by reflections on the faces of $C$. Define the graph $\Delta$ with a vertex for each face of $C$ and an edge between two vertices if they meet at a right angle.

$$
\Gamma=\left\langle r \in V(\Delta): r^{2}=1,(r s)^{2}=1 \text { if }(r, s) \in E(\Delta)\right\rangle
$$

where the generators $r$ are realized in $\operatorname{Isom}\left(\mathbb{H}^{n}\right)$ by reflections on the corresponding face.

Let $A$ be a subset of $V(\Delta)$ with induced subgroup $\Delta_{A}$ and $B$ the complement of $A$ in $V(\Delta)$ with induced subgraph $\Delta_{B}$. Let $\Gamma_{A}$ be the subgroup of $\Gamma$ generated by the elements of $A$, and similarly define $\Gamma_{B}$. Let $N\left(\Gamma_{B}\right)$ denote the normal closure of $\Gamma_{B}$ in $\Gamma$.

Lemma 4.6. $\Gamma / N\left(\Gamma_{B}\right)=\Gamma_{A}$, that is, $\Gamma_{A}$ is both a subgroup and a quotient of $\Gamma$. In particular, if $F$ is a hyperface of dimension $n-k \geq 2$ in $C$ and $\Gamma_{F}$ the reflection group of $F$ in $\operatorname{Isom}\left(\mathbb{H}^{n-k}\right)$, then $\Gamma$ retracts onto $\Gamma_{F}$.

Proof. Consider the group presentation

$$
\Gamma / N\left(\Gamma_{B}\right)=\left\langle r \in V(\Delta): r^{2}=1,(r s)^{2}=1 \text { if }(r, s) \in E(\Delta), t=1 \text { if } t \in V\left(\Delta_{B}\right)\right\rangle
$$

since if $t \in V\left(\Delta_{B}\right)$ then for any $r \in \Delta$, with $(r, t) \in E(\Delta)$, the relation $(r t)^{2}=1$ is equivalent in $\Gamma / N\left(\Gamma_{B}\right)$ to $r^{2}=1$, which we already had. Therefore,

$$
\begin{aligned}
\Gamma / N\left(\Gamma_{B}\right) & =\left\langle r \in V(\Delta)-V\left(\Delta_{B}\right): r^{2}=1,(r s)^{2}=1 \text { if }(r, s) \in E(\Delta)\right\rangle \\
& =\left\langle r \in V\left(\Delta_{A}\right): r^{2}=1,(r s)^{2}=1 \text { if }(r, s) \in E\left(\Delta_{A}\right)\right\rangle \\
& =\Gamma_{A} .
\end{aligned}
$$

From AVS93, we have the following facts:

- Any face of a hyperbolic right-angled polytope of dimension $n>2$ is a hyperbolic right-angled polytope of dimension $n-2$.

- Every $k$-dimensional face of a hyperbolic right-angled polytope belongs only to $n-k$ many hyperfaces.

In particular, any $(n-3)$-dimensional face (or vertex in the case $n=3$ ) belongs only to 3 hyperfaces. This also means that any two $(n-1)$ faces which intersect, must intersect in an $(n-2)$ face.

Now, suppose that $F$ is a face of $C$, i.e. a hyperplane of dimension $(n-1)$. Identify $H=\mathbb{H}^{n-1}$ with the hyperplane of $\mathbb{H}^{n}$ containing the face $F$. Let $r$ be the reflection on $H$, and hence on $F$. Let $F^{\prime}$ be a face of $C$ which meets $F$ at a right-angle. Then $r^{\prime}$, reflection on $F^{\prime}$, stabilizes $H$. Indeed, restricted to $H, r^{\prime}$ is a reflection on the face of $F$ where $F^{\prime}$ meets $F$.

Suppose $F_{1}$ and $F_{2}$ both meet $F$ and intersect at an $(n-3)$-dimensional face of $C$. By the facts above, $F_{1}$ and $F_{2}$ intersect at an $(n-2)$-dimensional face at a right-angle. Therefore for the corresponding reflections $r_{1}$ and $r_{2}$, we have that $\left(r_{1} r_{2}\right)^{2}=1$.

Let $S$ be the set of faces of $C$ which meet $F$ in $\mathbb{H}^{n}$ and not including $F$ itself. The map $\phi: \Gamma \rightarrow \Gamma_{S}$ given by

$$
r_{i} \mapsto \begin{cases}r_{i} & \text { if } F_{i} \in S \\ 1 & \text { if } F_{i} \notin S\end{cases}
$$

is a retraction. 
For a lower-dimensional hyperface (of dimension at least 2), repeat this process and compose to get a retraction from $\Gamma$ onto $\Gamma_{F}$.

Proof of Proposition 1.4. Each of the 2-dimensional faces of the hyperbolic Coxeter polyhefrom of dimension 6 with corresponding $\mathrm{RACG} \mathrm{SO}^{+}\left(F_{6} ; \mathbb{Z}\right)_{(2)}$ is a 2 dimensional hyperbolic Coxeter polygon with RACG SO${ }^{+}\left(F_{2} ; \mathbb{Z}\right)_{(2)}$ (refer to Theorem 2.1) . The intersection of the image of $\Delta_{m}$ (after $\varphi_{m}$ and conjugation by $A_{m}$ ) is exaclt

$$
\Delta_{m} \cap \operatorname{PSL}(2, \mathbb{Z})=\operatorname{PSL}(2, \mathbb{Z})_{(2)}=\left\langle\left(\begin{array}{ll}
1 & 2 \\
0 & 1
\end{array}\right),\left(\begin{array}{ll}
1 & 0 \\
2 & 1
\end{array}\right)\right\rangle
$$

a free group on 2 generators.

\section{Cocompact examples}

In this section we first consider a family of cocompact Kleinian known to virtually embed in a RACG. We then focus on a particular group in the family and a nonHaken Kleinian group in its commensurability class.

5.1. A family of cocompact Kleinian groups. Choose any positive prime $m \equiv$ $-1 \bmod (8)$ and let $Q_{m}^{\prime}$ be the quadratic form

$$
Q_{m}^{\prime}:=-m x_{1}^{2}+x_{2}^{2}+x_{3}^{2}+x_{4}^{2} .
$$

By Dirichlet's Theorem there are infinitely many such primes. For different $m$, the groups $\mathrm{SO}^{+}\left(Q_{m}^{\prime} ; \mathbb{Z}\right)$ are non-comensurable cocompact arithmetic lattices which all virtually embed in a RACG (see ALR01, Lemma 4.6(1),§6]).

Let $P_{m}^{\prime}$ be the quadratic form $-m x_{1}^{2}+x_{2}^{2}+x_{3}^{2}+x_{4}^{2}+m x_{5}^{2}$. Then $P_{m}^{\prime}=Q_{m}^{\prime} \oplus\langle m\rangle$. The group $\mathrm{SO}^{+}\left(Q_{m} ; \mathbb{Z}\right)$ is naturally a subgroup of $\mathrm{SO}^{+}\left(P_{m} ; \mathbb{Z}\right)$.

Lemma 5.1. The reduction modulo $m$ of an the first column of an element in $\mathrm{SO}^{+}\left(Q_{m}^{\prime} ; \mathbb{Z}\right)$ is $( \pm 1,0,0,0)$.

Proof. Suppose $N \in \pi_{m}\left(S O^{+}\left(Q_{m}^{\prime} ; \mathbb{Z}\right)\right)$. Then $N$ can be written as a block matrix

$$
\left(\begin{array}{ll}
N_{0} & N_{1} \\
N_{2} & N_{3}
\end{array}\right)
$$

where $N_{0}$ is a $1 \times 1$ matrix, $N_{1}$ is $1 \times 3, N_{2}$ is $3 \times 1$ and $N_{3}$ is $3 \times 3$. Since $N$ must satisfy $N^{t} \pi_{m}\left(S_{Q_{m}^{\prime}}\right) N=\pi_{m}\left(S_{Q_{m}^{\prime}}\right)$ we have

$$
\begin{aligned}
N^{t} \pi_{m}\left(S_{Q_{m}^{\prime}}\right) N & =\left(\begin{array}{ll}
N_{0}^{t} & N_{2}^{t} \\
N_{1}^{t} & N_{3}^{t}
\end{array}\right)\left(\begin{array}{cc}
0_{1 \times 1} & 0_{1 \times 3} \\
0_{3 \times 1} & I_{3 \times 3}
\end{array}\right)\left(\begin{array}{ll}
N_{0} & N_{1} \\
N_{2} & N_{3}
\end{array}\right) \\
& =\left(\begin{array}{ll}
N_{2}^{t} N_{2} & N_{2}^{t} N_{3} \\
N_{3}^{t} N_{2} & N_{3}^{t} N_{3}
\end{array}\right) \\
& \equiv\left(\begin{array}{ll}
0_{1 \times 1} & 0_{1 \times 3} \\
0_{3 \times 1} & I_{3 \times 3}
\end{array}\right) .
\end{aligned}
$$

Since $m$ is prime, $\mathbb{Z} / m \mathbb{Z}$ is a field and it must be that since $N_{2}^{t} N_{2} \equiv 0$, so $N_{2} \equiv 0_{1 \times 3}$. Therefore, the the first column of $N$ has form $(a, b m, c m, d m)^{t}$ and must satisfy $-m a^{2}+(m b)^{2}+(m c)^{2}+(m d)^{2}=-m$, equivalently $-a^{2}+m b^{2}+m c^{2}+m d^{2}=$ -1 . This implies $a^{2}=1 \bmod m$ and so $a= \pm 1 \bmod m$ and the first column is $( \pm 1,0,0,0)^{t}$ modulo $m$. 
Remark 5.2. An element $\mathrm{O}^{+}\left(Q_{m}^{\prime} ; \mathbb{Z}\right)$ is in $\mathrm{SO}^{+}\left(Q_{m}^{\prime} ; \mathbb{Z}\right)$ if the $(1,1)$-matrix entry is positive. Also, the group $\mathrm{SO}^{+}\left(Q_{m}^{\prime} ; \mathbb{Z}\right)$ contains elements whose first column reduces to $(-1,0,0,0)^{t}$. For example, when $m=7$,

$$
\left(\begin{array}{cccc}
6 & 1 & 2 & 0 \\
-7 & -2 & -2 & 0 \\
-14 & -2 & -5 & 0 \\
0 & 0 & 0 & 1
\end{array}\right) \in \mathrm{SO}^{+}\left(Q_{7}^{\prime} ; \mathbb{Z}\right)
$$

Let $\Delta^{m}$ be the index 2 subgroup of $\mathrm{SO}^{+}\left(Q_{m}^{\prime} ; \mathbb{Z}\right)$ of elements whose $(1,1)$-matrix entry is equivalent to 1 modulo $m$.

Proposition 5.3. Let $m=8 k-1$ be a positive prime number. The group $\Delta_{(2)}^{m}$ embeds in the $R A C G \mathrm{SO}^{+}\left(F_{4} ; \mathbb{Z}\right)_{(2)}$.

Proof. Note first that $m=(4 k)^{2}-(4 k-1)^{2}$, a difference of two squares. Consider the $5 \times 5$ matrix

$$
A_{m}^{\prime}=\left(\begin{array}{ccccc}
4 k & 0 & 0 & 0 & -(4 k-1) \\
0 & 1 & 0 & 0 & 0 \\
0 & 0 & 1 & 0 & 0 \\
0 & 0 & 0 & 1 & 0 \\
-(4 k-1) & 0 & 0 & 0 & 4 k
\end{array}\right)
$$

with inverse

$$
\left(A_{m}^{\prime}\right)^{-1}=\left(\begin{array}{ccccc}
\frac{4 k}{m} & 0 & 0 & 0 & \frac{4 k-1}{m} \\
0 & 1 & 0 & 0 & 0 \\
0 & 0 & 1 & 0 & 0 \\
0 & 0 & 0 & 1 & 0 \\
\frac{4 k-1}{m} & 0 & 0 & 0 & \frac{4 k}{m}
\end{array}\right)
$$

Let $S_{F}$ be the diagonal matrix associated to $F_{4}$ and $S_{P_{m}^{\prime}}$ the symmetric matrix associated to $P_{m}^{\prime}$ :

$$
S_{F}=\left(\begin{array}{ccccc}
-1 & 0 & 0 & 0 & 0 \\
0 & 1 & 0 & 0 & 0 \\
0 & 0 & 1 & 0 & 0 \\
0 & 0 & 0 & 1 & 0 \\
0 & 0 & 0 & 0 & 1
\end{array}\right) \text { and } S_{P_{m}^{\prime}}=\left(\begin{array}{ccccc}
-m & 0 & 0 & 0 & 0 \\
0 & 1 & 0 & 0 & 0 \\
0 & 0 & 1 & 0 & 0 \\
0 & 0 & 0 & 1 & 0 \\
0 & 0 & 0 & 0 & m
\end{array}\right) .
$$

Then $\left(A_{m}^{\prime}\right)^{t} S_{F} A_{m}^{\prime}=S_{P_{m}^{\prime}}$. Since $A_{m}^{\prime}$ has determinant $m$, it is in $\operatorname{GL}(5, \mathbb{Q})$. Therefore, the forms $F_{4}$ and $P_{m}^{\prime}$ are equivalent over $\mathbb{Q}$ and thus $A_{m}^{\prime} S O^{+}\left(P_{m}^{\prime} ; \mathbb{Q}\right)\left(A_{m}^{\prime}\right)^{-1}=$ $S O^{+}\left(F_{4} ; \mathbb{Q}\right)$ with $A_{m}^{\prime} S O^{+}\left(P_{m}^{\prime} ; \mathbb{Q}\right)\left(A_{m}^{\prime}\right)^{-1}$ and $S O^{+}\left(F_{4} ; \mathbb{Z}\right)$ commensurable.

By Lemma 5.1 a matrix $N$ in $\Delta_{(2)}^{m}$ sits naturally in $S O^{+}\left(P_{m}^{\prime} ; \mathbb{Z}\right)$ with form

$$
\left(\begin{array}{ccccc}
2 m a_{1}+1 & 2 b_{1} & 2 c_{1} & 2 d_{1} & 0 \\
2 m a_{2} & 2 b_{2}+1 & 2 c_{2} & 2 d_{2} & 0 \\
2 m a_{3} & 2 b_{3} & 2 c_{3}+1 & 2 d_{3} & 0 \\
2 m a_{4} & 2 b_{4} & 2 c_{4} & 2 d_{4}+1 & 0 \\
0 & 0 & 0 & 0 & 1
\end{array}\right)
$$

Then

$$
A_{m}^{\prime} N\left(A_{m}^{\prime}\right)^{-1}=
$$




$$
\left(\begin{array}{ccccc}
32 a_{1} k^{2}+1 & 8 k b_{1} & 8 k c_{1} & 8 k d_{1} & 32 k^{2} a_{1}-8 k a_{1} \\
8 k a_{2} & 2 b_{2}+1 & 2 c_{2} & 2 d_{2} & 8 k a_{2}-2 a_{2} \\
8 k a_{3} & 2 b_{3} & 2 c_{3}+1 & 2 d_{3} & 8 k a_{3}-2 a_{3} \\
8 k a_{4} & 2 b_{4} & 2 c_{4} & 2 d_{4}+1 & 8 k a_{4}-2 a_{4} \\
8 k a_{1}-32 k^{2} a_{1} & 2 b_{1}-8 k b_{1} & 2 c_{1}-8 k c_{1} & 2 d_{1}-8 k d_{1} & -32 a_{1} k^{2}+16 a_{1} k-2 a_{1}+1
\end{array}\right)
$$
is in $\mathrm{SO}^{+}\left(F_{4} ; \mathbb{Z}\right)_{(2)}$.

5.2. A non-Haken example. Commensurable with $\mathrm{SO}^{+}\left(Q_{7}^{\prime} ; \mathbb{Z}\right)$ and $\Delta_{(2)}^{7}$ is the fundamental group of a particular non-Haken hyperbolic 3-manifold mentioned in ALR01, p.616]. For this reason, we treat the case of $\mathrm{SO}^{+}\left(Q_{7}^{\prime} ; \mathbb{Z}\right)$ with more detail.

The arithmetic hyperbolic tetrahedral groups were studied in MR89. The tetrahedron $T_{6}$ of MR89 has dihedral angles $\left\{\frac{\pi}{2}, \frac{\pi}{3}, \frac{\pi}{4} ; \frac{\pi}{2}, \frac{\pi}{3}, \frac{\pi}{4}\right\}$ and volume approximately 0.2222287320 . Let $\Gamma$ be the index-2 subgroup of orientation preserving isometries in the reflection group for this tetrahedron. It is an arithmetic Kleinian group generated by the matrices

$$
\left\{\left(\begin{array}{cccc}
1 & 0 & 0 & 0 \\
0 & 0 & 1 & 0 \\
0 & 1 & 0 & 0 \\
0 & 0 & 0 & -1
\end{array}\right),\left(\begin{array}{cccc}
9 / 2 & 1 / 2 & 3 / 2 & 1 / 2 \\
-7 / 2 & 1 / 2 & -3 / 2 & -1 / 2 \\
-21 / 2 & -3 / 2 & -7 / 1 & -3 / 2 \\
7 / 2 & 1 / 2 & 3 / 2 & -1 / 2
\end{array}\right),\left(\begin{array}{cccc}
1 & 0 & 0 & 0 \\
0 & 0 & 0 & 1 \\
0 & 0 & 1 & 0 \\
0 & -1 & 0 & 0
\end{array}\right)\right\} .
$$

$\Gamma$ is commensurable with the group $\mathrm{SO}^{+}\left(Q_{7}^{\prime} ; \mathbb{Z}\right)$ generated by the matrices

$$
\left\{\left(\begin{array}{cccc}
1 & 0 & 0 & 0 \\
0 & 0 & 1 & 0 \\
0 & 1 & 0 & 0 \\
0 & 0 & 0 & -1
\end{array}\right),\left(\begin{array}{cccc}
1 & 0 & 0 & 0 \\
0 & 0 & 0 & 1 \\
0 & 0 & 1 & 0 \\
0 & -1 & 0 & 0
\end{array}\right),\left(\begin{array}{cccc}
6 & 1 & 2 & 0 \\
-7 & -2 & -2 & 0 \\
-14 & -2 & -5 & 0 \\
0 & 0 & 0 & 1
\end{array}\right)\right\} \text {. }
$$

It turns out that $\Gamma \cap \mathrm{SO}^{+}\left(Q_{7}^{\prime} ; \mathbb{Z}\right)$ is actually the group $\Delta^{7}$. It has index 3 in $\Gamma$ and index 2 in $\mathrm{SO}^{+}\left(Q_{7}^{\prime} ; \mathbb{Z}\right)$ and is generated by the matrices

$$
\begin{array}{r}
\left\{\left(\begin{array}{cccc}
1 & 0 & 0 & 0 \\
0 & 0 & 1 & 0 \\
0 & 1 & 0 & 0 \\
0 & 0 & 0 & -1
\end{array}\right),\left(\begin{array}{cccc}
1 & 0 & 0 & 0 \\
0 & 0 & 0 & 1 \\
0 & 0 & 1 & 0 \\
0 & -1 & 0 & 0
\end{array}\right),\left(\begin{array}{cccc}
8 & 2 & 2 & 1 \\
-14 & -3 & -4 & -2 \\
-14 & -4 & -3 & -2 \\
7 & 2 & 2 & 0
\end{array}\right),\right. \\
\\
\left.\quad\left(\begin{array}{ccccc}
8 & 0 & 3 & 0 \\
0 & 1 & 0 & 0 \\
-21 & 0 & -8 & 0 \\
0 & 0 & 0 & -1
\end{array}\right)\right\} .
\end{array}
$$

Magma shows that the reduction of $\Delta^{7}$ modulo 2 has order 24 , so $\left[\Delta^{7}: \Delta_{(2)}^{7}\right]=24$ and $\Gamma$ has a special subgroup of index 72 .

Both groups $\Gamma$ and $\mathrm{SO}^{+}\left(Q_{7}^{\prime} ; \mathbb{Z}\right)$ are contained in the same maximal arithmetic Kleinian group, (an image of) $\Gamma_{\mathcal{O}}$ where $\mathcal{O}$ is a maximal order in the invariant quaternion algebra (with notation as in [MR89]) with volume $\approx 0.1111143660$. It is shown in MR89 that $\Gamma^{(2)}$, the subgroup of $\Gamma$ generated by all the squares, is the group $\Gamma_{\mathcal{O}^{1}}=P \rho_{1}\left(\mathcal{O}^{1}\right)$.

There is a non-Haken hyperbolic 3-manifold obtained by a 4/1-Dehn filling on the once-punctured torus bundle with monodromy $R^{2} L^{2}$ (with the $R L$-factorization). It has volume $\approx 2.666744783$. Via Snap, one can check that its fundamental group, call it $\Theta$, is commensurable with $\Gamma$ because they have the same invariant arithmetic data. This group $\Theta$ is not contained in $\Gamma_{\mathcal{O}}$, but in some other maximal group. However, $\Theta^{(2)}$ is contained in $\Gamma_{\mathcal{O}^{1}}$ with index bounded above by 8 . Therefore, $\Theta$ has a special subgroup of index bounded above by $8 \cdot 72=576$. 


\section{ACKNOWLEDGEMENT}

The author would like to thank Yen Duong for asking a related question. The author also thanks Alan Reid for suggesting this problem and for his generous support, guidance, and encouragement.

\section{REFERENCES}

[Ago04] I. Agol. Tameness of hyperbolic 3-manifolds. arXiv:0405568, 2004. 22 pages, 1 figure.

[Ago08] Ian Agol. Criteria for virtual fibering. J. Topol., 1(2):269-284, 2008.

[Ago13] Ian Agol. The virtual Haken conjecture. Doc. Math., 18:1045-1087, 2013. With an appendix by Agol, Daniel Groves, and Jason Manning.

[ALR01] I. Agol, D. D. Long, and A. W. Reid. The Bianchi groups are separable on geometrically finite subgroups. Ann. of Math. (2), 153(3):599-621, 2001.

[AVS93] D. V. Alekseevskij, È. B. Vinberg, and A. S. Solodovnikov. Geometry of spaces of constant curvature. In Geometry, II, volume 29 of Encyclopaedia Math. Sci., pages 1-138. Springer, Berlin, 1993.

[BHW11] Nicolas Bergeron, Frédéric Haglund, and Daniel T. Wise. Hyperplane sections in arithmetic hyperbolic manifolds. J. Lond. Math. Soc. (2), 83(2):431-448, 2011.

[Can96] Richard D. Canary. A covering theorem for hyperbolic 3-manifolds and its applications. Topology, 35(3):751-778, 1996.

[CG06] Danny Calegari and David Gabai. Shrinkwrapping and the taming of hyperbolic 3manifolds. J. Amer. Math. Soc., 19(2):385-446, 2006.

[CLR97] D. Cooper, D. D. Long, and A. W. Reid. Essential closed surfaces in bounded 3manifolds. J. Amer. Math. Soc., 10(3):553-563, 1997.

[Dic01] L.E. Dickson. Linear Groups with an Exposition of the Galois Field Theory. B.G Teubner's Sammlung von Lehrbuchern auf dem Gebiete der mathematischen Wissenschaften mit Einschluss ihrer Anwendungen. B.G. Teubner, 1901.

[DJ00] Michael W. Davis and Tadeusz Januszkiewicz. Right-angled Artin groups are commensurable with right-angled Coxeter groups. J. Pure Appl. Algebra, 153(3):229-235, 2000.

[EGM98] J. Elstrodt, F. Grunewald, and J. Mennicke. Groups acting on hyperbolic space. Springer Monographs in Mathematics. Springer-Verlag, Berlin, 1998. Harmonic analysis and number theory.

[ERT12] Brent Everitt, John G. Ratcliffe, and Steven T. Tschantz. Right-angled Coxeter polytopes, hyperbolic six-manifolds, and a problem of Siegel. Math. Ann., 354(3):871-905, 2012.

[GM17] Daniel Groves and Jason Fox Manning. Quasiconvexity and Dehn filling. arXiv:1708.07968, 2017. 32 pages, 3 figures.

[HW08] Frédéric Haglund and Daniel T. Wise. Special cube complexes. Geom. Funct. Anal., 17(5):1551-1620, 2008.

[JM96] D. G. James and C. Maclachlan. Fuchsian subgroups of Bianchi groups. Trans. Amer. Math. Soc., 348(5):1989-2002, 1996.

[LR08] D. D. Long and A. W. Reid. Subgroup separability and virtual retractions of groups. Topology, 47(3):137-159, 2008.

[MR89] C. Maclachlan and A. W. Reid. The arithmetic structure of tetrahedral groups of hyperbolic isometries. Mathematika, 36(2):221-240 (1990), 1989.

[MR03] Colin Maclachlan and Alan W. Reid. The arithmetic of hyperbolic 3-manifolds, volume 219 of Graduate Texts in Mathematics. Springer-Verlag, New York, 2003.

[Ril75] Robert Riley. A quadratic parabolic group. Math. Proc. Cambridge Philos. Soc., 77:281$288,1975$.

[ST17] Jonathan Spreer and Stephan Tillmann. Unravelling the Dodecahedral Spaces. arXiv:1702.08080, 2017. 12 pages, 7 figures, 4 tables.

[Wis11] Daniel T. Wise. The structure of groups with quasiconvex hierarchy. 2011. 187 pages.

The University of Texas at Austin

E-mail address: mchu@math.utexas.edu 